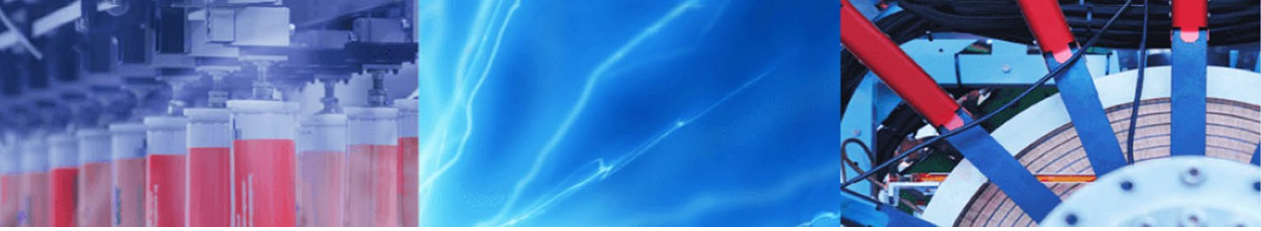

Research Article

\title{
Improving identification performance in iris recognition systems through combined feature extraction based on binary genetics
}

\author{
Mahdi Abbasi ${ }^{1}$ (D) \\ (c) Springer Nature Switzerland AG 2019
}

\begin{abstract}
The process of iris recognition is composed of several stages. The most important stage is feature extraction. The majority of systems use a single method of feature extraction. We used a binary genetic algorithm with a new fitness criterion to find an efficient combination of feature extraction methods with the aim of improving the performance of iris recognition systems. The proposed method makes use of a large number of filters and transforms which are widely used to extract iris features. The best combination of these techniques is found over iterations of the proposed algorithm. The final combination, which is argued to be the most efficient method of feature extraction, consists of a number of wavelet transforms, Gabor filter, and Fourier transform. ROC graphs are used to show the experimental superiority of the performance of this method over methods that use a single filter. According to our findings, the proposed method shows better performance in most situations. An FAR value of 0 and an FRR value of 0.092 was obtained by this method.
\end{abstract}

Keywords Iris · Identification · Feature extraction · Genetic algorithm

\section{Introduction}

Today there is an urgent need for reliable automatic identification devices. Computational methods for recognition of human physical features such as face, fingerprint, retina, voice, palm print, and eye are widely used for security, supervision, and ownership purposes [1, 2]. Most of the current methods, however, have serious limitations in real-world situations. Some of them require physical contact with the body, some need to take a sample, some require manual settings on the part of the user, and some methods impose high costs. Moreover, the features to be recognized should be both unique for individuals and invariable over time. Automatic or semi-automatic identification by means of physiological or behavioral features is referred to as biometrics. Examples of physiological features are fingerprints or facial images whereas behavioral features include one's actions such as signature or manner of speaking.
One of the methods that has recently received considerable attention is iris recognition. Due to the unique, abundant features of the complicated tissues of iris such as tracks, ridges, zigzag textures, rings, and patches, this method is more popular than other biometric methods [1-4] and results in more accurate identification. In addition, collection of samples is much easier than features such as DNA and, more importantly, iris is not easily changed over time (unlike voice tone) or by intentional illegal acts (e.g. burning with acid).

Feature extraction lies at the core of any identification system. On the one hand, the obtained feature vector should contain all the information about iris texture. On the other hand, its dimensions should be as small as possible because large vectors need large amounts of space for storage and, in addition, impose an extra computational burden on the system in the stages of extraction and matching.

After recognizing the inner and outer edges of the iris and mapping this area onto a square band with

Mahdi Abbasi, Abbasi@basu.ac.ir | 'Department of Computer Engineering, Engineering Faculty, Bu-Ali Sina University, Hamedan, Iran.

SN Applied Sciences (2019) 1:730 | https://doi.org/10.1007/s42452-019-0777-9 
dimensions of $512 * 64$, a feature vector should be extracted from this band. The vector should make use of the unique features of iris texture. In fact, it should only encode the most important pieces of information so that comparison between the input image and the stored codes could be done easily and successfully. The majority of identification systems produce appropriate codes by analyzing the information of the middle band of iris texture in the frequency range. The efficiency of a system is defined as the balance between False Acceptance Rate (FAR) and False Rejection Rate (FRR). The former refers to the number of individuals who were wrongly identified and the latter refers to the number of individuals who exist in the database but were not identified.

In this paper we propose a binary genetic algorithm to find an efficient combination of features with the aim of improving the performance of iris recognition system. The proposed method selects from a large number of filters and transforms which are widely used in the literature to extract iris features. Our algorithm finds the best combination of these techniques over a number of acceptable iterations.

The next section is a brief review of previous work in the field of biometric identification systems. Then the method and data used in the present study will be described and discussed in detail. Afterwards, the present method will be discussed in terms of performance and compared with other methods. The final section provides some concluding remarks and suggestions for further research.

\section{Related work}

Identification based on iris images consists of the analysis of features that exist in the pigmented tissue around the pupil. The complicated tissues of the iris include remarkable features such as tracks, ridges, zigzag textures, rings, and patches [5]. Iris is scanned through an ordinary camera without any close contact with the person. This brings about the advantage of privacy and comfort in comparison with methods like scanning the retina that need physical contact with the camera. Iris textures vary from one person to another [6] and it has been asserted that iris is more unique than fingerprint [7].

Many image-processing techniques are used to extract unique features from the iris and transform images to biometric codes. A biometric code is obtained from applying mathematical operations to an image. The obtained codes are stored in a database and when a user is to be identified, the image of their iris is encoded so as to be matched with the codes in the database. The system searches the code with the smallest difference. If the difference is smaller than a certain threshold, the person is identified. Identification systems based on iris images have recently received considerable attention, mainly through the work of John Daugman at Cambridge University [8]. Another system with a high rate of success is Wildes Iris Recognition System [9]. This system did not make any error when tested on 520 images. Another system is Lim which showed a success rate of 98.4 percent for 6000 images [10]. Results obtained from different biometric identification methods lead us to the conclusion that iris recognition is the most reliable method of identification [11]. The major problem with regard to this method is lack of sufficiently large databases of iris images, which reduces the generalizability of the results. In addition, most experiments make use of high-quality images.

A new algorithm developed by Azizi et al. [12] performs iris recognition by means of non-linear approximation coefficients and transform. Their study utilizes Daugman's method to segment and normalize iris images. In the extraction stage, only the middle band of the normalized images are analyzed by contourlet transform. This method is more accurate than wavelet transform method because contourlet transform has a relatively higher ability to capture directional information.

Alvarez et al. [13] proposed a powerful method of key points-based feature extraction under variable quality conditions. Their method acts on the basis of efficient fusion of three information sources of SIFT features at an identical level. It functions through a proposed method of fusion which is based on three well-known measurement units, namely, AUC, EER, and CRR. The innovation of this method lies in its use of the potential of SIFT features to describe and fuse key points in order to improve the performance of non-ideal iris images. Analyses show that, when combined, these information sources are extremely powerful. The efficiency of this method decreases with the reduction of image resolution. Although feature extraction in this algorithm requires a longer time than in other algorithms, its matching is much faster. Therefore, it is suitable for real-time identification.

Abikoye et al. [14] proposed a new feature extraction method using fast wavelet transform (FWT). In order to encode iris features, this system transforms iris image from Cartesian to polar coordinate system and then extracts features using FWT. This algorithm has a high speed and a low complexity rate.

Sankar et al. [15] developed a region-based method for extracting iris features. This method uses triplet half-band filter bank (THFB) for multi-resolution analysis. This type of analysis can be performed on any two-dimensional signal and extract the features of its texture. The study was conducted on three libraries, i.e. CASIv3, UBIRISv3, and IITD. The findings suggest that this technique is more accurate than previous techniques. 
Another method proposed by Ashok et al. [16] makes use of the power of three classification types based on k-nearest distance, sparse display, and genetic algorithm. The experiments show that the false error rate (FER) of this method approximates is very low.

Recently, several methods like [17-27] have exploited convolution neural networks (CNNs) and deep learning methods to enhance the performance of the iris recognition systems via precise segmentation of the iris image or dense extraction of useful features. However, without optimal selection of the features that maximize the discrimination the high computational complexity and memory requirement of such methods lead to considerable challenges in their implementation.

All of the reviewed methods and recent methods lack from the efficient combination of the different feature extraction methods which can considerably enhance the performance of iris recognition system $[2,3,28]$. In the next section a new method is explained which aims to resolve this problem.

\section{Proposed method for iris recognition}

The proposed algorithm makes use of Libor Masek iris recognition system. This system has an automatic segmentation unit based on Hough transform and is equipped with the ability to detect the ring of iris, the pupil region, eyelids and eyelashes, and light reflection. In the first step of preprocessing, the edges of the iris are separated from the remaining parts of the input eye image. The inner edge of the iris which is connected to the pupil and the outer edge which is connected to the sclera are detected by means of Canny algorithm. The points on these circular edges are then determined by the parametric equation of Hough transform.

The general stages of performing the identification process using iris images are as depicted in Fig. 1.

Iris recognition consists of capturing the image, separating the iris, normalization, feature extraction, and matching. In addition to the iris, the captured images also contain pupil, eyelids, eyelashes, and light reflections in the eye. For a better performance, these parts must be separated from the main texture of the iris.
Fig. 1 A schematic view of an identification system based on iris recognition

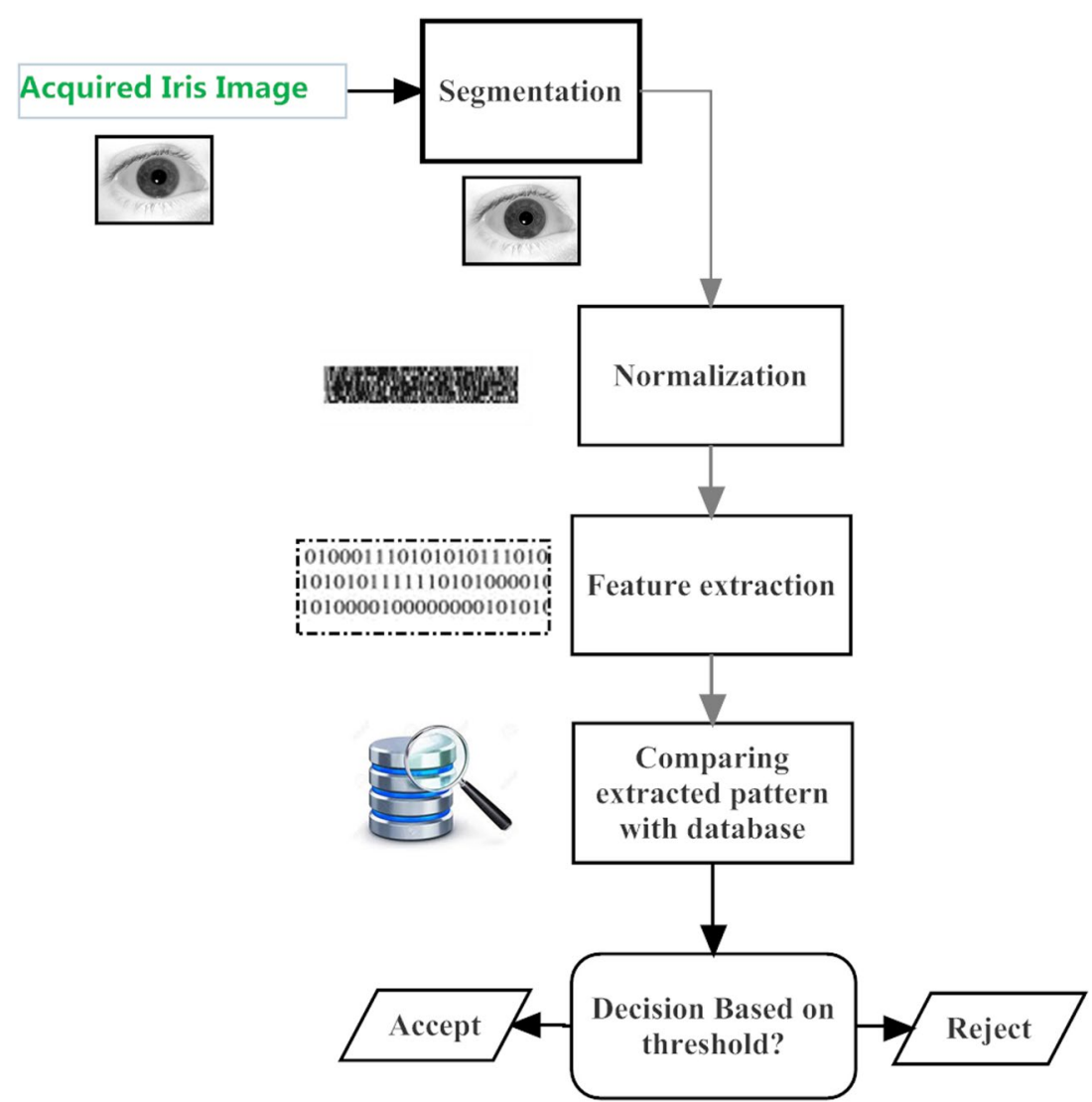

SN Applied Sciences 


\subsection{The database of eye images}

To identify the image with iris images, we must first have an image of the eye. We use a database of human eye images which is named CASIA [29]. Figure 2 shows a sample image form this database. The pupil has lower intensity as than other areas.

\subsection{Segmentation}

The purpose of this section is to find the iris boundaries, border of sclera and iris and the border of pupil. Next, this process finds the areas of iris which are blocked by the eyelids. Eyelids usually cover the upper and lower parts of the iris.

The success of this stage depends on the quality of the images obtained from the eyes of the people. Existing images in the database of CASIA are not reflexive in pupil and iris due to the use of infrared light in imaging. But, in the LEI database images, because of using visible light for imaging, reflection problem is observed [30]. In addition, in people whose iris have a dark color, if you use the visible light to capture the image, an unapparent difference would be present in the intensity of the light of iris and pupil which makes the separation of these two parts so difficult. Segmentation is an important step in the identification system. By an inaccurate segmentation system, the images with incorrectly detected boundaries reduce the system's success rate.

In Daugman's method, integral operator is used to recognize iris edges and the eyelids are separated by means of two arcs. Differential integration can be considered as the differences of Hough transform because the first derivative of the image is used for searching [31].

Hough transform is a method of feature extraction used in image analysis, computer vision, and image processing. This method aims at finding the instances of a pattern in an image. The instances may be imperfect and partly distorted [32]. An example of the uses of this method is to find a straight line in an image. Hough transform, therefore, is an algorithm that can be used to recognize specified shapes in an image. In order to recognize a shape through Hough transform, the image must have a certain parametric form. This is why this transform is usually used to find shapes like lines, circles, or parabolas. To detect a circle in the image, first we should use an edge detection algorithm such as Canny or Sobel to find the edges and then we use circular Hough transform to find the radius and central point of pupil and iris [33].

\subsection{Normalization}

The obtained region of iris is normalized within a square block with fixed dimensions in order to overcome the problem of the varying size of iris. Here, we deploy the commonly used Daugman's methods to normalize the iris images [31]. Daugman's suggested model which is cited in most papers, is indeed a transformation process. Each point in the iris region is mapped to a pair $(r, \theta)$ in the polar coordinate system, where $r$ reassembles the radial distance in range $[0,1]$, and the $\theta$ is in the range $[0,2 \pi]$.

When the iris region is separated from the eye image, the next stage is the transfer of the iris area to a coordinate with constant dimensions that can be easily compared. The discrepancy between the dimensions of the eye images is usually due to the iris being stretched in the images. This may be caused by the pupil enlargement due to changes in ambient light. Other reasons would also be involved including the variable distance of the eyes from the camera, rotation of the camera lens, head rotation, and eye rotation in the eye bowl.

The normalization process rebuilds the iris area so that two pictures taken in different situations of an eye could be easily compared by converting the iris areas to the same two-dimensional areas. Another point is that the pupil, depending on the position of the nose in the face, is not concentric with the iris [34]. This case is also considered during normalization of the iris image. Figure 3 illustrates the result of the normalization on a sample iris image.
Fig. 2 Two sample image of eye from CASIA dataset
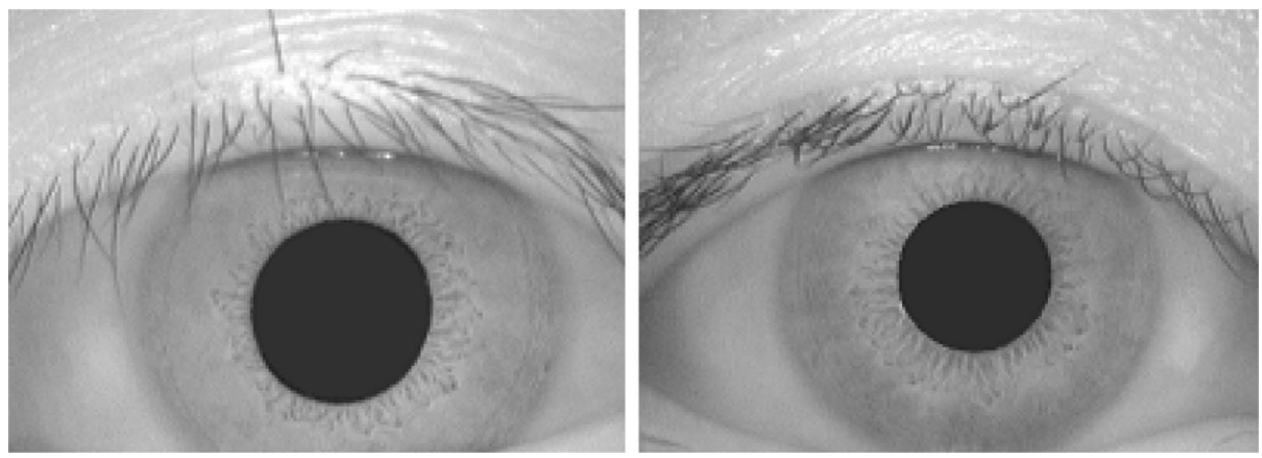
Fig. 3 a Image of the iris; $\mathbf{b}$ result of Hough transform; c example of a normalized image

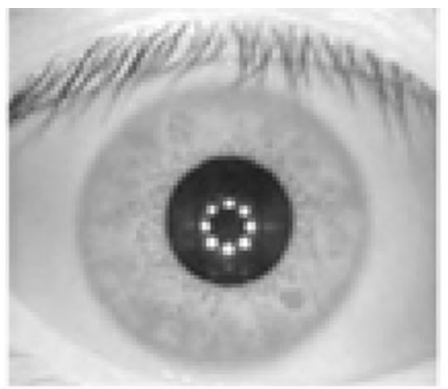

(a)

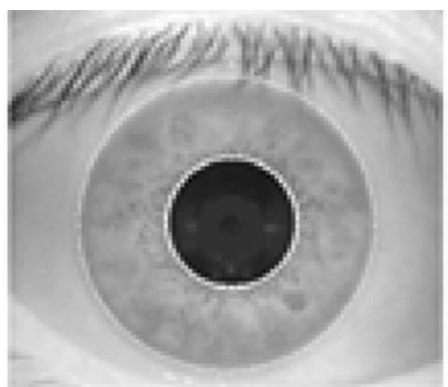

(b)

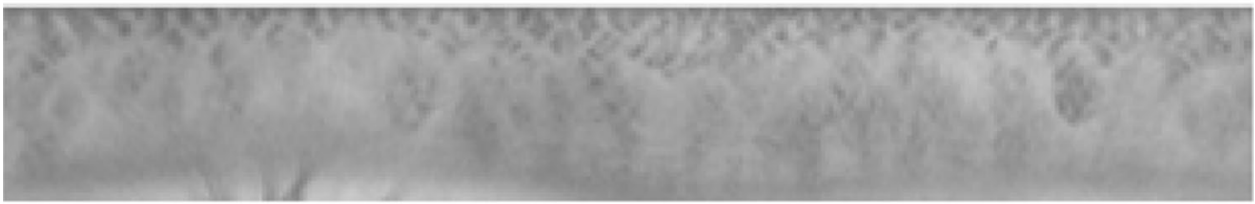

(c)

\subsection{Extraction of the Features of Iris}

In this step, the features of iris texture are extracted and used for creation of a biometric sample. We have made use of four feature extraction methods in our work: wavelet decomposition, Laplacian mask, Gabor transform, and Fourier transform. The filters used in our method are shown in Table 1.

In addition to Gabor filters [35], our method uses several other feature extraction methods that have been previously used in extracting the efficient features of iris and combines them to develop the optimum method with the highest performance. In what follows, we address these methods and discuss how the best combination of them can be obtained through genetic algorithm.

There plausible reasons behind using these four types of filter. Fourier transform is a filter with low computational cost and provides us with a possibility to use image phase information. It has so far been widely used in for iris recognition. Laplacian filters allow for more details of the image and are capable of sharpening the image. They have also been applied to identification based on iris recognition in a number of studies [36]. Gabor filters can be used as directional and scale-adjustable detectors to detect lines and edges in an image. The statistic features of Gabor transform can be used to determine the structure and visual content of images. Gabor filters have already been applied to image analysis, texture segmentation, and image recognition. It is the only type of filter used for feature extraction in Libor Masek system [37].

In the present study, Gabor filter was used with a bandwidth of 0.5 and a central frequency of 0.0556 . Wavelets are a group of mathematical functions which are used to decompose continuous signals into their frequency components. In these functions, the resolution of each component is equal to its scale. The decomposition wavelet transform of each function is defined on the basis of its functions. Wavelets (usually known as daughter wavelets) are transformed and scaled instances of a function (called mother wavelet) with finite length and extremely damped oscillation. In comparison with Fourier transform, wavelet transform has a higher ability to be localized. All these filters have recently become very popular in the field of iris recognition [1-4, 38, 39]. Following is a brief description of two commonly used methods for extracting features from a normalized image of iris.

\subsubsection{Wavelet transform}

Image representation is a fundamental issue in image processing research. Good representation of images has a direct influence on the results of any procedure of image processing. Among the variety of representation methods, multi-scale representations are the most desirable because they allow a controlled flexibility in designing methods for automatically analyzing and deriving information from image data. Discrete Wavelet Transform (DWT) analysis of images is a powerful multi-scale representation method. This method produces better spatial and spectral localization of image signal compared with other multi-scale representations such as Gaussian and Laplacian pyramid. The result of applying this transform to image signal consists of two complementary signals, namely, approximation and details. The former is a representation of low-frequency parts of image and the latter is a representation of highfrequency parts of image [16]. Therefore, repetitive application of DWT decomposes the image signal into different sub-bands so that the lower-frequency sub-bands have 
Table 1 Types of feature extraction in the proposed method

\begin{tabular}{|c|c|c|}
\hline ID & Type & Description \\
\hline 1 & Haar wavelet & Horizontal details of decomposition \\
\hline 2 & Haar wavelet & Vertical details of decomposition \\
\hline 3 & Haar wavelet & Diagonal details of decomposition \\
\hline 4 & Daubechies 2 wavelet & Horizontal details of decomposition \\
\hline 5 & Daubechies 2 wavelet & Vertical details of decomposition \\
\hline 6 & Daubechies 2 wavelet & Diagonal details of decomposition \\
\hline 7 & Symlet 2 wavelet & Horizontal details of decomposition \\
\hline 8 & Symlet 2 wavelet & Vertical details of decomposition \\
\hline 9 & Symlet 2 wavelet & Diagonal details of decomposition \\
\hline 10 & Coiflet 1 wavelet & Horizontal details of decomposition \\
\hline 11 & Coiflet 1 wavelet & Vertical details of decomposition \\
\hline 12 & Coiflet 1 wavelet & Diagonal details of decomposition \\
\hline 13 & Biorthogonal 1.1 wavelet & Horizontal details of decomposition \\
\hline 14 & Biorthogonal 1.1 wavelet & Vertical details of decomposition \\
\hline 15 & Biorthogonal 1.1 wavelet & Diagonal details of decomposition \\
\hline 16 & Reverse biorthogonal 1.1 wavelet & Horizontal details of decomposition \\
\hline 17 & Reverse biorthogonal 1.1 wavelet & Vertical details of decomposition \\
\hline 18 & Reverse biorthogonal 1.1 wavelet & Diagonal details of decomposition \\
\hline 19 & Meyer wavelet & Horizontal details of decomposition \\
\hline 20 & Meyer wavelet & Vertical details of decomposition \\
\hline 21 & Meyer wavelet & Diagonal details of decomposition \\
\hline 22 & Biorthogonal 2.6 wavelet & Vertical details of decomposition \\
\hline 23 & Biorthogonal 2.6 wavelet & Diagonal details of decomposition \\
\hline 24 & Biorthogonal 2.6 wavelet & Horizontal details of decomposition \\
\hline 25 & Reverse biorthogonal 2.6 wavelet & Horizontal details of decomposition \\
\hline 26 & Reverse biorthogonal 2.6 wavelet & Vertical details of decomposition \\
\hline 27 & Reverse biorthogonal 2.6 wavelet & Diagonal details of decomposition \\
\hline 28 & Gabor filter & Real part of filter output \\
\hline 29 & Gabor filter & Imaginary part of filter output \\
\hline 30 & Laplacian filter & Filter output \\
\hline 31 & Fourier transform & Real part of filter output \\
\hline 32 & Fourier transform & Imaginary part of filter output \\
\hline
\end{tabular}

finer frequency resolution and coarser time resolution compared to the higher-frequency sub-bands.

\subsubsection{Gabor filters}

Gabor functions are example of wavelet filters which are widely used in a variety of applications of computer vision such as texture analysis and edge detection. In all such applications, it is necessary to analyze the spatial frequency components of an image in a localized manner. For localized frequency analysis of an image signal, it is necessary to have a Gaussian envelope whose width adjusts with the frequency of the complex sinusoids. Hence, the convolution kernel of Gabor elementary filters are Gaussians modulated by complex sinusoids. Consequently, the Gabor filters could optimally achieve joint resolution/localization in space and spatial frequency domains. As a result, configured appropriately and accurately, Gabor filters have a good performance in detecting textures and edges [35]. Here, phase data are extracted from the one-dimensional Log-Gabor filter and quantized at four levels. Figure 4 shows a sample feature vector extracted from a normalized iris image.

In order to standardize the iris pattern in a binary biometric format, hamming distance is used for matching iris patterns. Two patterns are matched correctly if their distance is smaller than a certain threshold. Any change in the threshold value will change the values of FAR and FRR which stand

01000010111101010010101111010100101011010101010100 10100111000111101110100010011000110110110001011110

Fig. 4 A sample feature vector extracted form a normalized iris image 
in a negative relationship, i.e. increasing one would decrease the other. The former stands for false acceptance rate and is computed as:

$F A R=\frac{\text { Total False Acceptance }}{\text { Total False Attemps }}$

The FAR parameter shows the probability that the system incorrectly matches the input pattern to a non-matching template in the database. It measures the percent of invalid inputs that are incorrectly accepted. The latter stands for false rejection rate and is computed as:

$F R R=\frac{\text { Total False rejection }}{\text { Total True Attemps }}$

The FRR parameter shows the the probability that the system fails to detect a match between the input pattern and a matching template in the database. It measures the percent of valid inputs that are incorrectly rejected.

As lower amounts of FAR and FRR errors are a sign of better performance in feature extraction and ultimately iris recognition, Eq. 3 is used as fitness function. The search algorithm seeks to decrease this value. In the next stage, production of the second generation helps to find solutions with the smallest values of fitness function.

Fitness $=\alpha \times F A R+\beta \times F R R$

In Eq. 3, $\alpha$ and $\beta$ are constants that are determined manually and indicate the importance of the rate of their corresponding errors. The flowchart of the calculation of fitness function is shown in Fig. 5.

To reduce computation complexity, first the features are extracted from all images and stored in a four-dimensional matrix (The firs dimension for image ID, the second for type of extraction, and the third and fourth for the features). Then selections are made from among different features by using the chromosome. As with the computational cost, the optimum combination is found only once, and that is where the main computational load lies. During the experimental phase, only optimum features are extracted and, therefore, the computational costs are not high. For calculation of fitness, all the images in the database compared in pairs and FAR and FRR are calculated through Eqs. 1 and 2. Finally, by Eq. 1 we can obtain the fitness of the chromosome, i.e. the combination of filters
Fig. 5 The flowchart of the calculation of fitness function for each chromosome

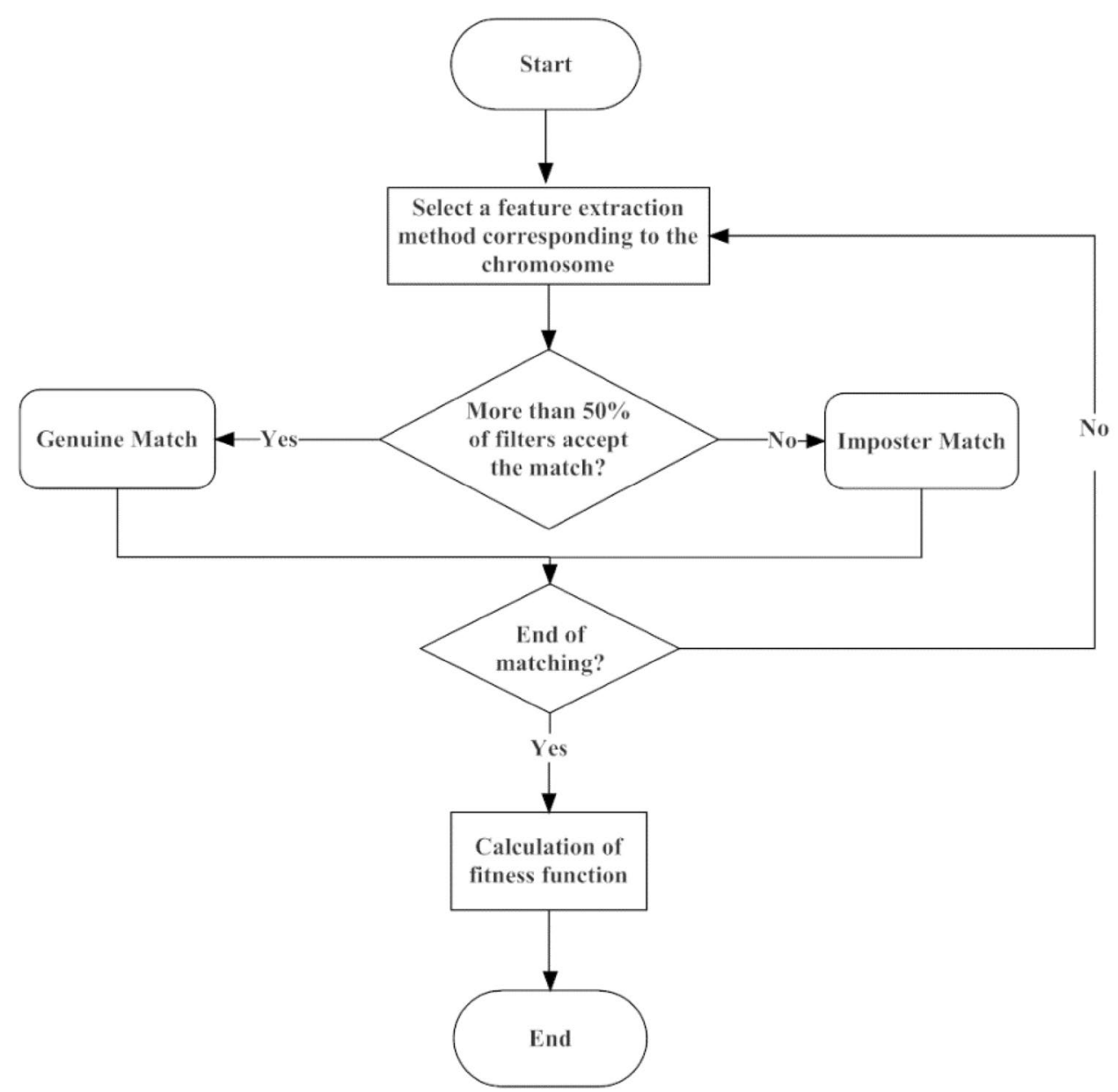


in feature extraction. Figure 6 depicts the flowchart of the proposed algorithm.

\subsection{Matching}

The obtained patterns of iris texture in the previous stage are now compared with Hamming's mean differential distance

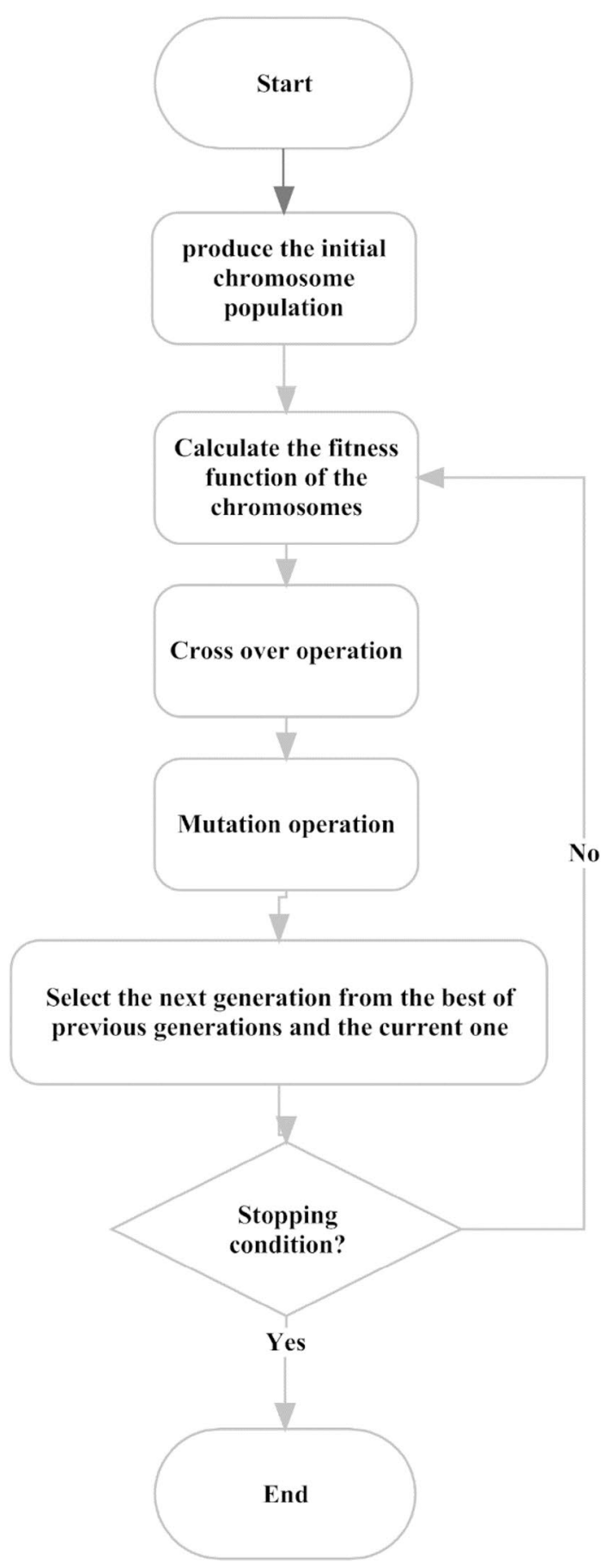

Fig. 6 The flowchart of the proposed algorithm which, in a normalized state, gives a score between 0 and 0.5. If the score is smaller than the determined threshold, the iris pattern can be identified as valid; otherwise, it is rejected as an invalid pattern. In information theory, the Hamming distance between two strings of equal length is the number of positions at which the corresponding symbols are different. Since the output of feature extraction was a binary matrix, the Hamming distance between two iris images with a single method of feature extraction equals the number of elements that contain different bits.

\subsection{Data and performance analysis}

This section briefly describes the database used in this study and the method of iris recognition is explained in detail and compared with other methods. Also, Libor Masek iris recognition system is briefly described. Since we have used genetic algorithm to find near-optimal solutions, this algorithm is described in detail.

\subsection{The database}

In contrast to other biometric methods which enjoy a large number of databases for experiments, there are only limited data available for iris images. The present study uses CASIA V.1 for the main experiments due to its high-quality images. In addition, we can compare the efficiency of our proposed method with other methods. The images were captured by infrared light and stored in BMP format with the dimension of $320 \times 280[13,29]$. In order to obtain the best filters for feature extraction and maximize recognition capability, we used the iris images of 50 persons and for each person 7 images were selected. This makes a total number of 350 image, resulting in $350 \times 7=2450$ genuine matches and $350 \times 343=120050$ imposter matches. Figure 7 shows some of typical images from CASIA V1.0 database.

In addition to this database, MNU V.2 database of the Multimedia University of Malaysia which contains iris images of persons from Asia, Middle East, Africa, and Europe. This database is made up of 995 images from 100 individuals [40].

The next database to be used was UBIRIS 1. It contains 1877 images of the right eye of 241 Europeans and has been prepared in three versions [41]. For this study, the $200 \times 150$ grayscale version was used.

\section{Evaluation and experiments}

This section is a description of the method of obtaining the best combined filter by the proposed method and finding a near-optimal solution (i.e. chromosome). The 


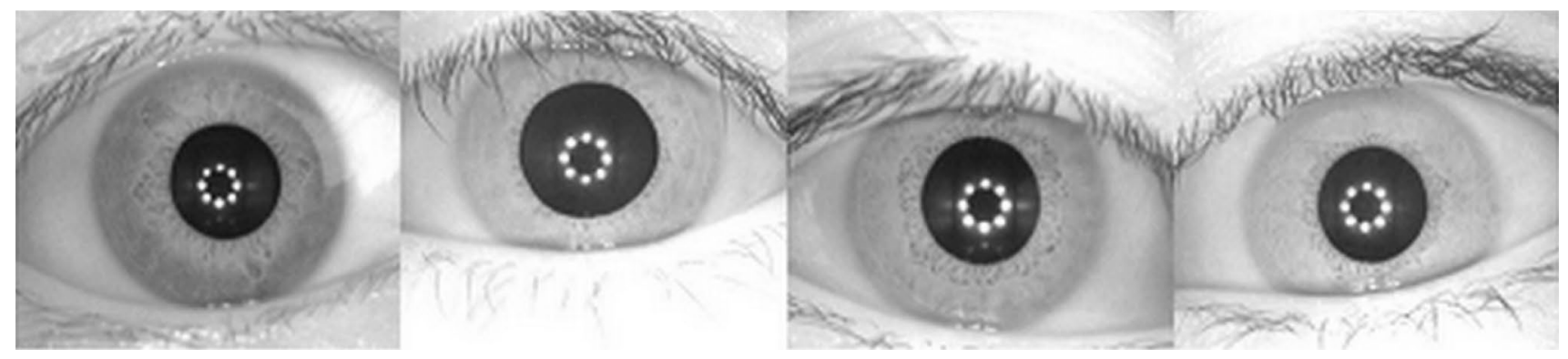

Fig. 7 Sample images from CASIA V1.0

experiments were performed on CASIA V1.0 and replicated on the other two databases. After obtaining the best combination of filters, the performance of the combined filter was compared with the filters that were already known to have the best performance in iris recognition.

Biometric systems are usually assessed by the True Accept Rate (TAR), where two biometric samples are properly decided to be the same person and the True Reject Rate (TRR) where the system correctly decided that two samples are not the identical person. The False Accept Rate (FAR) is defined as the rate where people are accepted as the same identity while in reality they are different and False Reject Rate (FRR) is where two samples from the same person are rejected. The TRR and FRR can be computed as FRR =1-TAR and TRR =1-FAR, respectively. The TAR and FAR are normally obtained by varying an acceptance decision threshold.

In this paper, the comparison is done with the help of Receiver Operating Characteristic (ROC) graph. The ROC curve is a plot of TAR against FAR for all possible operating points [3]. The best performance belongs to the biometric system that has the largest area under the curve (a maximum of 1). The descriptions of the graphs show that the filter obtained from the proposed algorithm has a better performance than the previous filters.

As shown in Eq. (3), there are two constants in the fitness function which have to be set manually. However, as FAR value is too small (approximately $1 / 1000$ times) in comparison with FRR rate (on the pre-specified standard threshold $\lambda=0.37$ ) and the value of FAR is crucial to the system's performance in iris recognition, the two constants were set as $\alpha=1000$ and $\beta=1$ in all experiments (an iris recognition system is expected to have an FAR rate close to 0 because this parameter shows false acceptance rate and is more important than FRR in most applications).

First, the proposed algorithm calculates the fitness function of the initial population. The value of this function for each chromosome is computed according to Eq. (3). In each iteration, the best filters are selected for producing the next generation. The optimization algorithm will be ended if, for 10 consecutive iterations, none of the produced filters has a better performance than the best filter produced in the previous iteration. Another condition to stop the algorithm is to go through a certain number of iterations, which was set to 1000 . Obviously, the highest amount of fitness is 0 and if a chromosome is found with this fitness value, it will be meaningless to continue the algorithm.

\section{Performance evaluation}

Changing the parameters of the genetic algorithm will change the fitness value. We seek the optimum state which is the lowest fitness value. In Fig. 8, the fitness of the system is examined for different crossover probabilities to obtain the optimum value. Another important parameter of genetic algorithm is mutation probability. With increase in this parameter, the amount of scanning in the algorithm will increase. Therefore, different solutions will be found by the algorithm which may be better or worse than those of the parents. The effect of this parameter on fitness is described in the following.

Figure 10 depicts the process of obtaining the best filter by using the proposed algorithm. It should be noted that, in the implementation of genetic algorithm, the crossover probability was taken as 0.8 and the mutation probability was taken as 0.3 (Figs. 8, 9). Moreover, single-point crossover was used. It means that 80 percent of the best chromosomes are combined in a way that two chromosomes are broken on a random point and linked to produce a new chromosome. To select chromosomes for crossover, Tournament selection operator was used. In this type of selection, a subset of a population is selected and the members of this subset compete with each other. Finally, only one individual is selected from each subset for production. In our proposed method, the number of members in each set was taken as 3 .

As can be seen in Fig. 10, the proposed algorithm has a progressive performance in obtaining more appropriate 


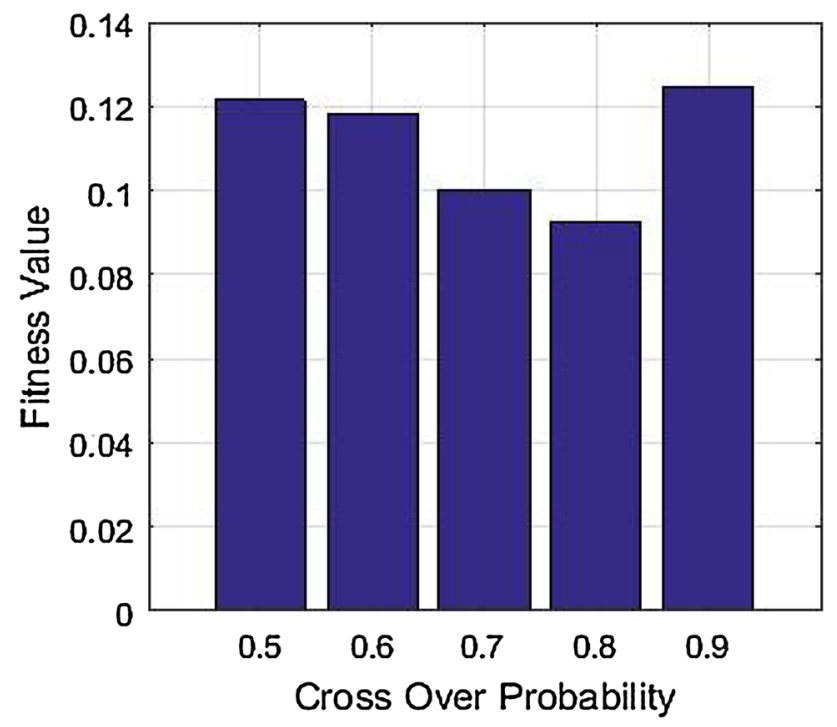

Fig. 8 Fitness value for different crossover possibilities

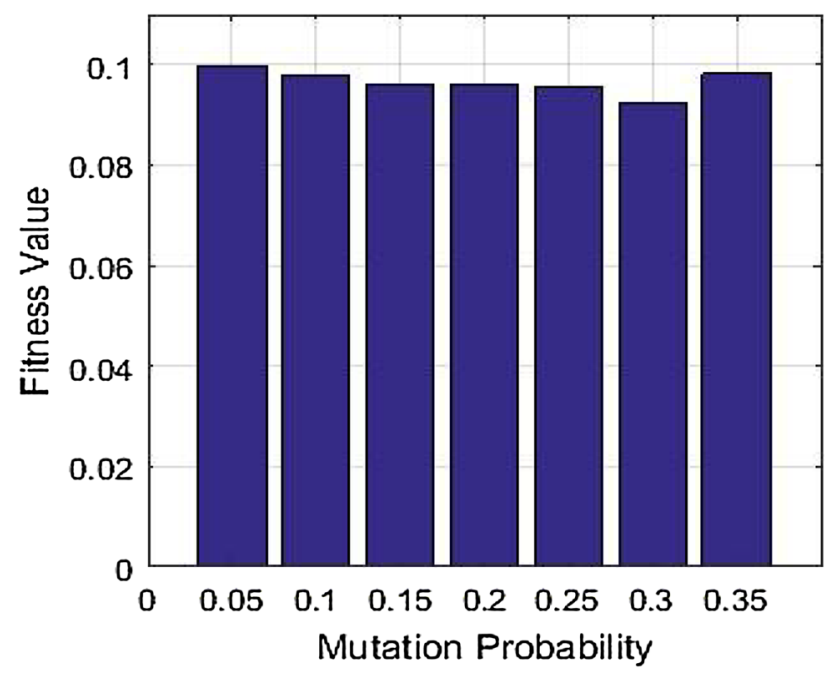

Fig. 9 Fitness value for different mutation probabilities

solutions and, finally, the fitness value remains constant between iterations 195 and 205, thereby stopping the algorithm.

Figure 11 shows the best chromosome found in CASIA V1.0. With a fitness value of 0.092517006802721 , this chromosome has the smallest fitness value. This value was obtained with an FAR value of 0 and an FRR value of 0.092517006802721 , which shows that the system had no false acceptance and the error rate of rejections was less than 10 percent.

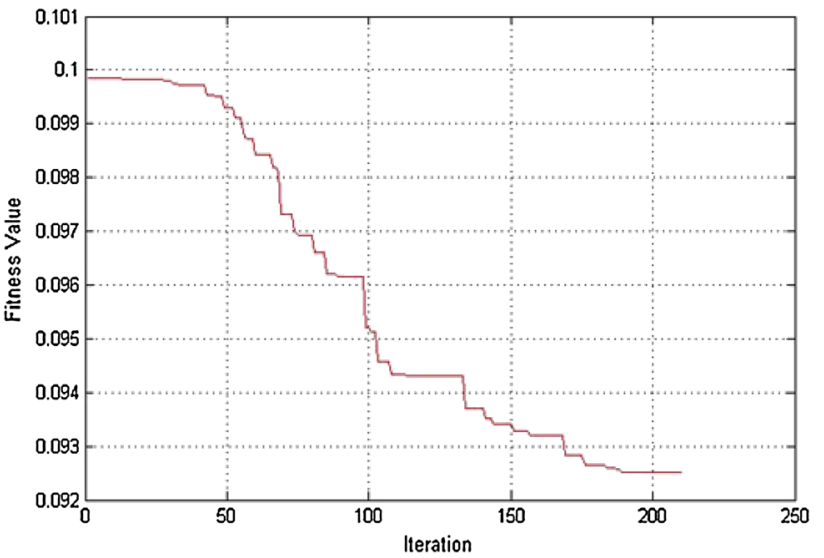

Fig. 10 The process of obtaining near-optimal solution through the proposed algorithm

Given the chromosome in Fig. 11 and the filters in Table 1, the combination of filters for feature extraction in the proposed method includes the horizontal decomposition details of Haar wavelet, the vertical decomposition details of Daubechies 2 wavelet, the vertical decomposition details of Symlet 2 wavelet, the diagonal decomposition details of Symlet 2 wavelet, the vertical decomposition details of Meyer wavelet, the vertical decomposition details of Biorthogonal 2.6 wavelet, the vertical decomposition details of Reverse Biorthogonal 2.6 wavelet, the imaginary part of Gabor filter, and the imaginary part of Fourier transform. The following graphs were plotted for CASIA V1 by means of this feature extraction method. It should be noted that the proposed method creates a unique chromosome for each database.

Following is a comparison between the performance of the obtained filter combination and that of Haar wavelet filter and Gabor filter which are widely known to have an acceptable performance in iris recognition in Libor Masek system. The comparison was made through ROC graphs. As shown before, this graph can be drawn by using the different threshold values in the stage of matching. In fact, the parameters of TAR (True Acceptance Rate) and FAR will be calculated according to the changes in the threshold and used to draw this graph. Figure 12 shows the ROC graph of these three filters to compare their performance in the iris recognition system.

As can be seen in Fig. 12, the proposed filter performs better than all other filters in all performance items. As a result, it can be inferred that the proposed filter combination is superior to all single filters used in iris recognition in Libor Masek. 
Following is a comparison between our implemented system and a number of other methods discussed in [8].

Figure 13 indicates the great performance of the proposed method tested on CASIA V.1 as compared with recent methods. Figures 14 and 15 respectively represent the results of the previous experiment as repeated with the data of MNU V.2 and UBIRIS1 databases. It can be seen that this method shows a better performance with this database, which is more obvious in higher values of FAR. With the third set of data, too, the proposed method clearly outperforms other methods for all FAR values. In Fig. 16, the area under the ROC curves of the experiments on different databases again shows that the proposed method has a better performance.

\section{Conclusion and suggestions}

Our proposed method aimed at finding a combination of filters to improve the total performance of the system. For this purpose, we used binary genetic algorithm to be able to select the best combination of different wavelet transforms, Fourier transform, and Gabor filter. Evaluations and comparisons showed that the proposed method has

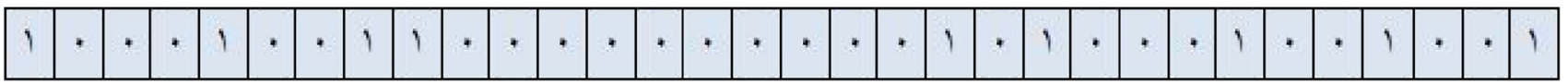

Fig. 11 The best chromosome found

Fig. 12 ROC graph for Gabor filter, wavelet filter, and the propose filter combination
Fig. 13 ROC graph for three iris recognition systems, i.e. Fast-Hessian, Weighted sum rule and the proposed method, tested on CASIA V1
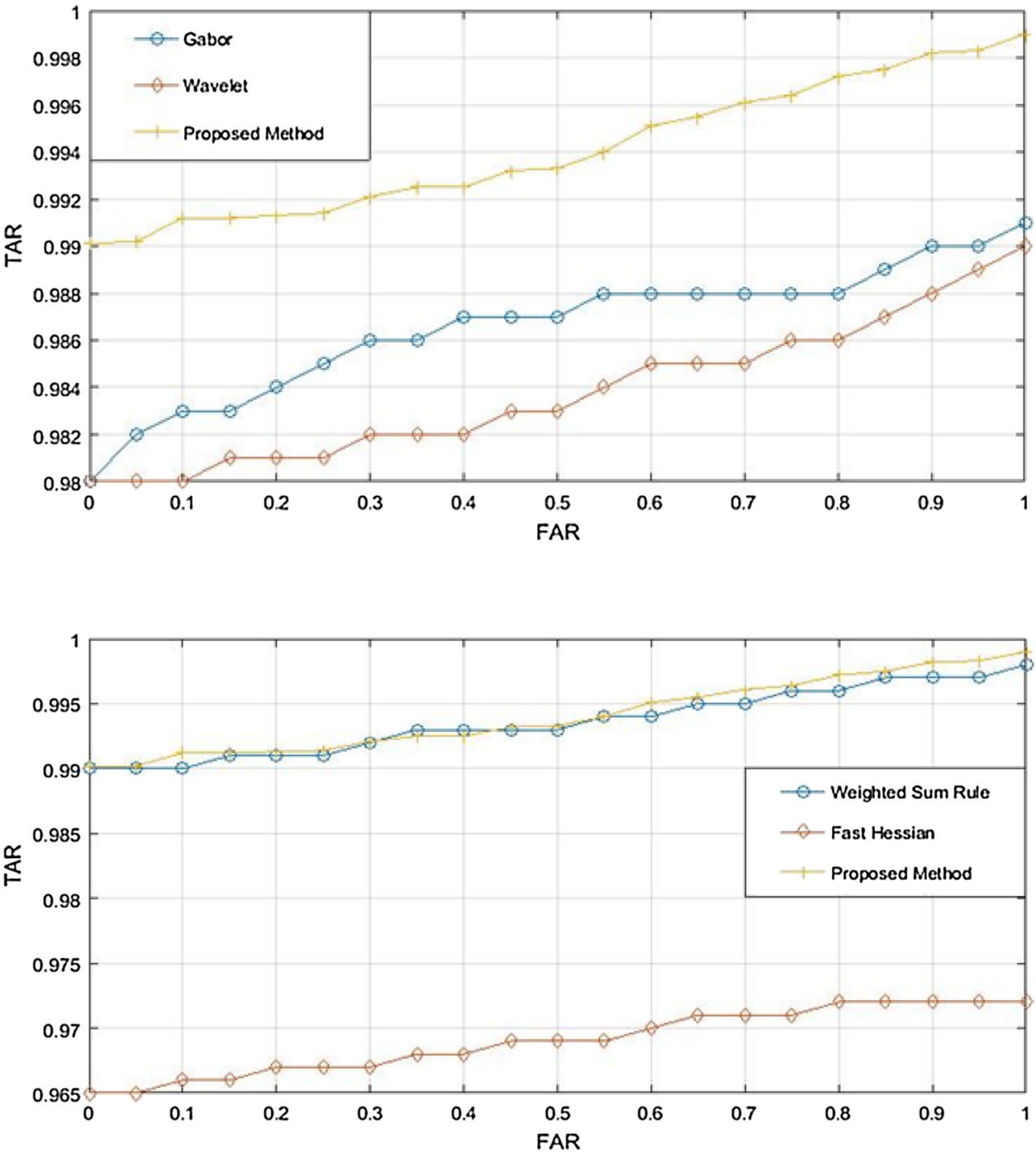

SN Applied Sciences 
Fig. 14 ROC graph for three iris recognition systems, i.e. Fast-Hessian, Weighted sum rule and the proposed method, tested on MNU V.2
Fig. 15 ROC graph for three iris recognition systems, i.e. Fast-Hessian, Weighted sum rule and the proposed method, tested on UBIRIS
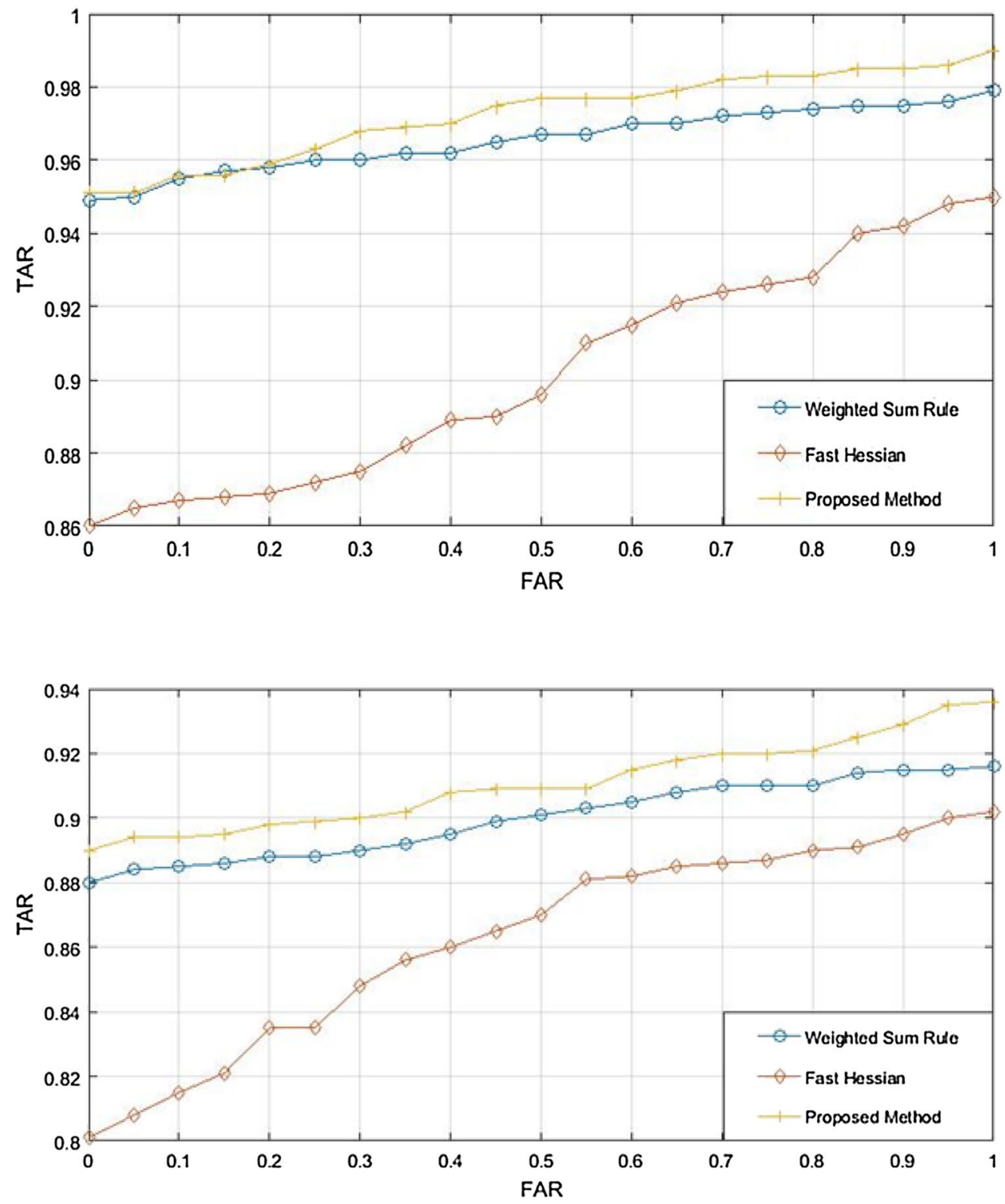

advantages over other methods of feature extraction that are used in iris recognition systems.

The reduction of error rate as a result of increase iteration in the proposed algorithm leads us to conclude that increasing the iterations as well as further configuration of the initial parameters of genetic algorithm may bring about more accurate results in future works.
Recently deep learning methods and convolutional neural networks are considered as powerful tools for segmentation and feature extraction of the biometric images $[4,17,18,20,26]$. They also accelerate the extraction of many diverse features from segmented biometric images in a small time $[1,21]$. This power would highly boost our iris recognition system. 
Fig. 16 The area under the ROC curves of the systems for different databases

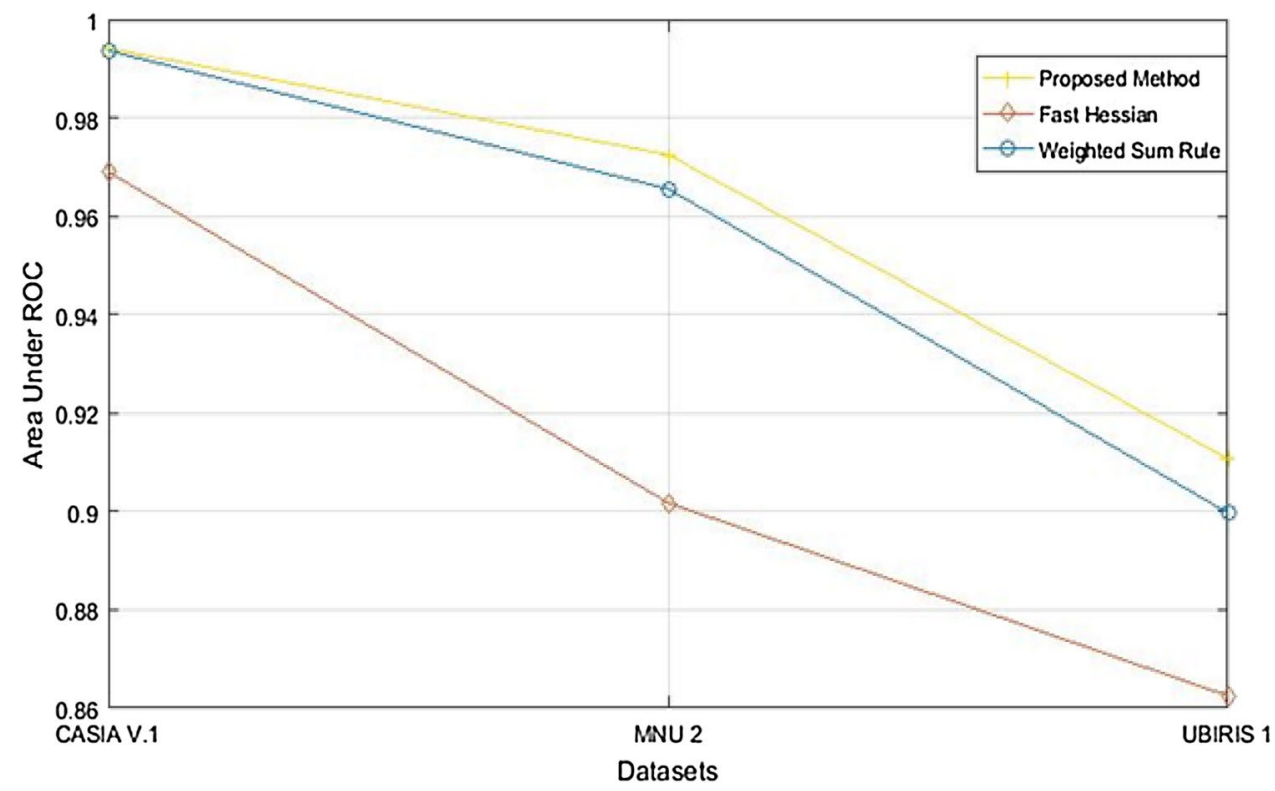

\section{Compliance with ethical standards}

Conflict of interest On behalf of all authors, the corresponding author states that there is no conflict of interest.

\section{References}

1. Nazmdeh V, Mortazavi S, Tajeddin D, Nazmdeh H, Modarresi Asem M (2019) Iris recognition; from classic to modern approaches, pp 981-988

2. Lozoya-Santos JJ, Sepúlveda-Arróniz V, Tudon-Martinez JC, Ramirez-Mendoza RA (2019) Survey on biometry for cognitive automotive systems. Cognit Syst Res 55:175-191

3. Soleimani SA, Asem MM (2019) Iris live detection assessment; a structural survey. In: 2019 IEEE 9th annual computing and communication workshop and conference (CCWC), pp 0974-0980

4. Harakannanavar SS, Prashanth C, Kanabur V, Puranikmath VI, Raja K (2019) An extensive study of issues, challenges and achievements in iris recognition. Asian J Electr Sci 8:25-35

5. Davision $\mathrm{H}$ (1962) The eye. Academic, Landon

6. Ma L, Wang Y, Tan T (2002) Iris recognition using circular symmetric filters. In: Object recognition supported by user interaction for service robots, pp 414-417

7. Jain AK, Bolle R, Pankanti S (2006) Biometrics: personal identification in networked society, vol 479. Springer, Berlin

8. Daugman J, Downing C (2001) Epigenetic randomness, complexity and singularity of human iris patterns. Proc R Soc Lond Ser B Biol Sci 268:1737-1740

9. Wildes RP (1997) Iris recognition: an emerging biometric technology. Proc IEEE 85:1348-1363

10. Sung H, Lim J, Park J-H, Lee $Y$ (2004) Iris recognition using collarette boundary localization. In: Proceedings of the 17th international conference on pattern recognition, 2004. ICPR 2004, pp 857-860

11. Sanderson S, Erbetta J (2000) Authentication for secure environments based on iris scanning technology, pp 8-9

12. Azizi A, Pourreza HR (2009) A new method for iris recognition based on contourlet transform and non linear approximation coefficients. In: International conference on intelligent computing, pp 307-316

13. Alvarez-Betancourt Y, Garcia-Silvente M (2016) A keypointsbased feature extraction method for iris recognition under variable image quality conditions. Knowl-Based Syst 92:169-182

14. Abikoye Oluwakemi C, Sadiku J, Adewole Kayode S, Jimoh Rasheed G (2014) Iris feature extraction for personal identification using fast wavelet transform (FWT). Structure 6:1-5

15. Barpanda SS, Majhi B, Sa PK (2015) Region based feature extraction from non-cooperative iris images using triplet half-band filter bank. Opt Laser Technol 72:6-14

16. Bhateja AK, Sharma S, Chaudhury S, Agrawal N (2016) Iris recognition based on sparse representation and k-nearest subspace with genetic algorithm. Pattern Recognit Lett 73:13-18

17. Zhao Z, Kumar A (2019) A deep learning based unified framework to detect, segment and recognize irises using spatially corresponding features. Pattern Recognit 93:546-557

18. Zhao T, Liu Y, Huo G, Zhu X (2019) A deep learning iris recognition method based on capsule network architecture. IEEE Access 7:49691-49701

19. Wang K, Kumar A (2019) Cross-spectral iris recognition using CNN and supervised discrete hashing. Pattern Recognit 86:85-98

20. Ribeiro $E$, Uhl A, Alonso-Fernandez F (2019) Iris super-resolution using CNNs: is photo-realism important to iris recognition? IET Biom 8:69-78

21. Nguyen K, Fookes C, Sridharan S (2019) Constrained design of deep iris networks. arXiv preprint arXiv:1905.09481

22. Liu M, Zhou Z, Shang P, Xu D (2019) Fuzzified image enhancement for deep learning in iris recognition. IEEE Trans Fuzzy Syst. https://doi.org/10.1109/TFUZZ.2019.2912576

23. Gangwar A, Joshi A, Joshi P, Raghavendra R (2019) DeeplrisNet2: learning deep-IrisCodes from scratch for segmentation-robust visible wavelength and near infrared iris recognition. arXiv preprint arXiv:1902.05390

24. Noh, S-I, Bae K, Park Y, Kim J (2003) A novel method to extract features for iris recognition system. In: Kittler J, Nixon MS (eds) Audio- and video-based biometric person authentication. AVBPA 2003. Lecture notes in computer science, vol 2688. Springer, Berlin, pp 862-868 
25. Akhundov R, Saxby DJ, Edwards S, Snodgrass S, Clausen P, Diamond LE (2019) Development of a deep neural network for automated electromyographic pattern classification. J Exp Biol 222:jeb198101

26. Bazrafkan S, Corcoran P (2018) Enhancing iris authentication on handheld devices using deep learning derived segmentation techniques. In: 2018 IEEE international conference on consumer electronics (ICCE), pp 1-2

27. Al-Waisy AS, Qahwaji R, Ipson S, Al-Fahdawi S, Nagem TA (2018) A multi-biometric iris recognition system based on a deep learning approach. Pattern Anal Appl 21:783-802

28. De Marsico M, Petrosino A, Ricciardi S (2016) Iris recognition through machine learning techniques: a survey. Pattern Recognit Lett 82:106-115

29. NLPR (2015) National Laboratory of Pattern Recognition, Casia, P.R.C (1 ed.). http://www.nlpr.ia.ac.cn/en/. Accessed 11 July 2018

30. Barry C, Ritter N (1999) Database of 120 greyscale eye images. Lions Eye Institute, Perth Western Australia

31. Daugman J (2009) How iris recognition works. In: Bovik Al (ed) The essential guide to image processing. Elsevier, Amsterdam, pp 715-739

32. Forsyth D, Ponce J (2002) Computer vision: a modern approach. Prentice Hall Professional Technical Reference, Upper Saddle River

33. Masek L (2003) Recognition of human iris patterns for biometric identification. Master's thesis, University of Western Australia

34. Daugman J, Downing C (2001) Epigenetic randomness, complexity and singularity of human iris patterns. Proc R Soc Lond B Biol Sci 268:1737-1740

35. Kadri F, Meraoumia A, Bendjenna H, Chitroub S (2016) Palmprint \& iris for a multibiometric authentication scheme using
Log-Gabor filter response. In: 2016 international conference on information technology for organizations development (IT4OD), pp 1-5

36. Proenca H, Alexandre LA (2007) Toward noncooperative iris recognition: a classification approach using multiple signatures. IEEE Trans Pattern Anal Mach Intell 29:607-612

37. Misiti M, Misiti Y, Oppenheim G, Poggi J-M (2013) Wavelets and their applications. Wiley, Hoboken

38. Pandey M (2016) An amalgamated strategy for iris recognition employing neural network and hamming distance. In: Satapathy S, Mandal J, Udgata S, Bhateja V (eds) Information systems design and intelligent applications. Springer, Berlin, pp 739-747

39. Minaee $S$, Abdolrashidi A, Wang $Y$ (2015) Iris recognition using scattering transform and textural features. In: 2015 IEEE signal processing and signal processing education workshop (SP/SPE), pp 37-42

40. Alvarez-Betancourt $Y$, Garcia-Silvente $M$ (2014) An overview of iris recognition: a bibliometric analysis of the period 2000-2012. Scientometrics 101:2003-2033

41. Miyazawa K, Ito K, Aoki T, Kobayashi K, Nakajima H (2008) An effective approach for iris recognition using phase-based image matching. IEEE Trans Pattern Anal Mach Intell 30:1741-1756

Publisher's Note Springer Nature remains neutral with regard to jurisdictional claims in published maps and institutional affiliations. 